MATHEMATICS OF COMPUTATION

Volume 72, Number 244, Pages 1711-1730

S 0025-5718(03)01533-3

Article electronically published on May 1, 2003

\title{
V-CYCLE CONVERGENCE OF SOME MULTIGRID METHODS FOR ILL-POSED PROBLEMS
}

\author{
BARBARA KALTENBACHER
}

\begin{abstract}
For ill-posed linear operator equations we consider some V-cycle multigrid approaches, that, in the framework of Bramble, Pasciak, Wang, and $\mathrm{Xu}$ (1991), we prove to yield level independent contraction factor estimates. Consequently, we can incorporate these multigrid operators in a full multigrid method, that, together with a discrepancy principle, is shown to act as an iterative regularization method for the underlying infinite-dimensional ill-posed problem. Numerical experiments illustrate the theoretical results.
\end{abstract}

\section{INTRODUCTION}

Consider the first kind operator equation

$$
\mathbf{T} x=y,
$$

where $\mathbf{T}: \mathbf{X} \rightarrow \mathbf{Y}$ is a compact linear operator between Hilbert spaces $\mathbf{X}$ and $\mathbf{Y}$, and $y \in \mathbf{R}(\mathbf{T})\left(+\mathbf{R}(\mathbf{T})^{\perp}\right)$, which we suppose to model some linear or linearized inverse problem. Numerous applications leading to inverse problems appear in science and industry. Their mathematical formulation (11) typically leads to an illposed problem in the sense that the range of $\mathbf{T}$ is nonclosed, i.e., the (generalized, cf., e.g., [10]) inverse of $\mathbf{T}$ is unbounded. Therefore, given data $y^{\delta}$ with arbitrary small noise $\delta$ in

$$
\left\|y-y^{\delta}\right\| \leq \delta,
$$

one can possibly arrive at large deviations in the solution when using conventional numerical methods. Hence special stable approximation methods for solving (1) so-called regularization methods (cf. [10, [11], 23], 24], 26], [30], 32])- have to be applied, the most well known one certainly being Tikhonov regularization:

$$
\tilde{x}:=\left(\mathbf{T}^{*} \mathbf{T}+\alpha I\right)^{-1} \mathbf{T}^{*} y^{\delta} .
$$

As a consequence of the ill-posedness, any numerical approximation method for (11) converges - if at all - in general arbitrarily slowly as the noise level $\delta$ goes to zero, and convergence rates can only be obtained under additional regularity or sourcewise representation conditions

$$
x^{\dagger} \in \mathbf{R}\left(\left(\mathbf{T}^{*} \mathbf{T}\right)^{\mu}\right),
$$

Received by the editor November 21, 2000 and, in revised form, April 11, 2002.

2000 Mathematics Subject Classification. Primary 65J20, 65R30, 65N55.

Key words and phrases. Ill-posed problem, multigrid methods.

The author was supported by the Fonds zur Förderung der wissenschaftlichen Forschung under grant T 7-TEC and project F1308 within Spezialforschungsbereich F013.

(C)2003 American Mathematical Society 


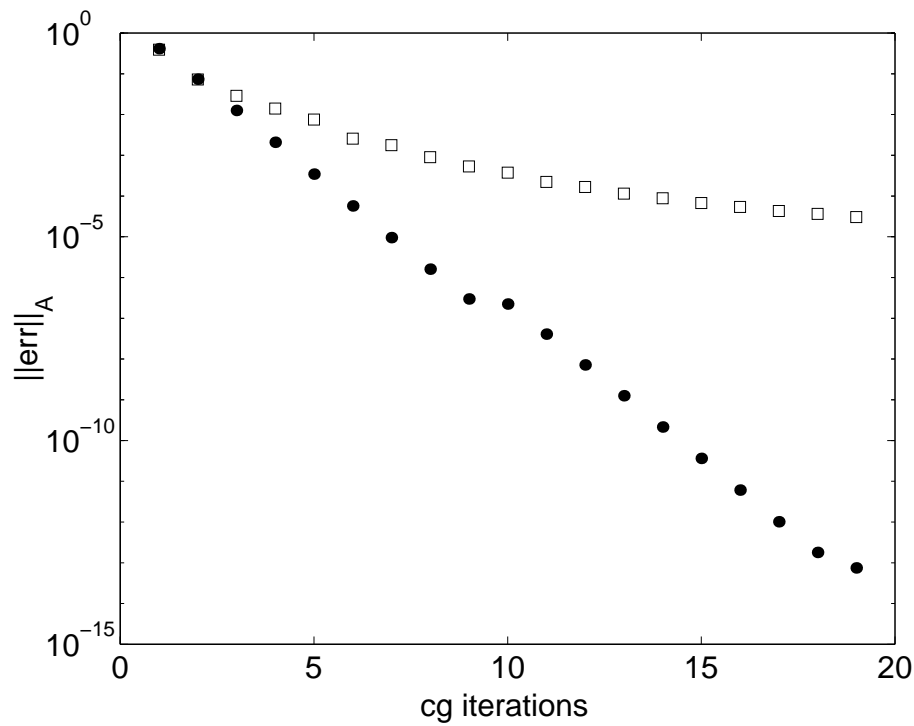

Figure 1. Error development over the first twenty iterations of a multigrid preconditioned conjugate gradient method for the Abel integral equation (see Section [5 below for details) with Gauß-Seidel smoother (squares) and with smoother as proposed in [22] (dots).

where $\mu>0$ is a real exponent and the operator $\left(\mathbf{T}^{*} \mathbf{T}\right)^{\mu}$ is defined in the sense of functional calculus (cf., e.g., Section 2.3 in [10]). The under condition (4) optimal convergence rates for regularized approximations $\tilde{x}$ to $x^{\dagger}$ are

$$
\left\|\tilde{x}-x^{\dagger}\right\|=O\left(\delta^{\frac{2 \mu}{2 \mu+1}}\right)
$$

and therefore are always slower than the rate $O(\delta)$ that is typical for well-posed problems.

While multigrid methods (MGM) are already well established as extremely efficient solvers for large scale systems of equations originating from the discretization of partial differential or second kind integral equations (see, e.g., 1], 2], 4], [6], [7, 14]), the situation is quite different for first kind integral equations, or, generally, ill-posed problems (11), though. This is due to the adverse structure of the singular systems of compact operators (high frequency eigenfunctions correspond to small singular values) that foils the smoothing properties of schemes such as Gauß-Seidel iteration, used as smoothers in MGM for well-posed problems; see Figure 1

Applications of MGM to (Tikhonov-)regularized ill-posed problems can be found in [15], 29. While these papers analyze MGM for equations with small but nonvanishing regularization parameter $\alpha>0$ in (3), we are more interested in the situation that the regularization is solely due to discreteness (see [9, 12], 16], [17, [25], [28, [31]), so without introducing any additional (possibly artificial) regularization term. For this purpose, MGM based on a smoother proposed by King in [22] have turned out to be appropriate; see Figure 1 Further contraction number estimates for these MGMs as well as an analysis of the full MGM with a priori or a posteriori stopping rule as a regularization method for solving the original infinite-dimensional ill-posed problem (11), including a generalization to nonlinear problems, can be found in [18] 
and [21]. Since the theory presented there still needs at least alternating or Wcycles, the aim of the present paper is to give V-cycle convergence proofs of MGM for ill-posed problems. To this end, we follow the approach of [3], where a V-cycle convergent multigrid method for pseudo-differential operators of order minus one is presented and analyzed, and we show how it can be used for defining and analyzing V-cycle MG algorithms for general equations (1). Here norm equivalence theorems such as those from 27] (with their computational implementation as given, e.g., in [5]) play an important role. Based on these norm estimates, the smoother by King 22. can also be modified in such a way that V-cycle convergence can be proved; see Section 3

The level-independence of the contraction factors in the proposed multigrid operators makes it possible to use them in a full multigrid method for the iterative solution of the underlying infinite-dimensional problem (1). In combination with an a posteriori stopping rule that finds the optimal balance between approximation error and propagated data noise, this can be shown to define a convergent and order optimal regularization method for the ill-posed operator equation (1); see Section 4

Section $[5$ reports on numerical experiments with the proposed methods and is supposed to illustrate the foregoing theoretical results.

In the following we will use the notation $c$ or $C$ for positive constants that are typically "small" or "large" and can have different values whenever they appear.

\section{Discretization}

Discretization of the ill-posed problem (11). Finite-dimensional problems, though they might be ill-conditioned, are always well-posed in the sense of stable data dependence of a solution (as long as its existence and uniqueness can be guaranteed, which can be done, e.g., by using a best approximate solution concept). This fact forms the basis for several finite-dimensional projection approaches for the regularization of ill-posed operator equations (10). Note, however, that for ill-posed problems, discretization in preimage space does not generically yield a convergent approximation, even in the noiseless situation (cf. the counterexample due to Seidman cited in 10]). Therefore we prefer here the approach of discretization in image space $(9,12,25,31)$, defined by projecting (1), with possibly noisy data $y^{\delta}$ satisfying (21), onto finite-dimensional nested subspaces $\mathbf{Y}_{n}$ of $\mathbf{Y}$, whose union is dense in $\mathbf{Y}$ :

$$
\mathbf{Y}_{1} \subseteq \mathbf{Y}_{2} \subseteq \cdots \subseteq \mathbf{Y} \wedge \overline{\bigcup_{n \in \mathbb{N}} \mathbf{Y}_{n}}=\mathbf{Y}
$$

The best approximate solution of this projected equation lies in the finite-dimensional space

$$
\mathbf{X}_{n}:=\mathbf{T}^{*} \mathbf{Y}_{n}
$$

so with

$$
\mathbf{P}_{n}:=\operatorname{Proj} \mathbf{X}_{n}, \quad \mathbf{Q}_{n}:=\operatorname{Proj}_{\mathbf{Y}_{n}}^{\mathbf{X}},
$$

we arrive at an appropriate discretization of (1)

$$
\mathbf{Q}_{n} \mathbf{T} x_{n}=\mathbf{Q}_{n} y^{\delta}, \quad x_{n} \in \mathbf{X}_{n},
$$


or, equivalently

$$
\begin{gathered}
x_{n}=\mathbf{T}^{*} u, \quad u \in \mathbf{Y}_{n}, \\
\left\langle\mathbf{T}^{*} u, \mathbf{T}^{*} v\right\rangle_{\mathbf{X}}=\left\langle y^{\delta}, v\right\rangle_{\mathbf{Y}}, \quad \text { for all } v \in \mathbf{Y}_{n}
\end{gathered}
$$

Indeed, the exact solution of (77) can be seen as an approximation of the exact best approximate solution $x^{\dagger}=\mathbf{T}^{\dagger} y$ of (11) since, in the noiseless case $\delta=0$, we have

$$
x_{n}=\mathbf{P}_{n} x^{\dagger} .
$$

Nonvanishing data noise is in the worst case amplified by the factor $\underline{\gamma}_{n}^{-1}$, where $\underline{\gamma}_{n}$ is the smallest singular value of the system matrix in (7), i.e.,

$$
\underline{\gamma}_{n}:=\frac{1}{\left\|\left(\mathbf{Q}_{n} \mathbf{T}\right)^{\dagger} \mathbf{Q}_{n}\right\|}=\inf _{v \in \mathbf{X}_{n}} \frac{\left\|\mathbf{Q}_{n} \mathbf{T} v\right\|}{\|v\|},
$$

that, by the compactness of $\mathbf{T}$, goes to infinity as $n \rightarrow \infty$, i.e., with increasing refinement of the discretization we have to face a possibly unboundedly growing propagated data noise contribution to the total error - this reflects the instability of the underlying infinite-dimensional problem. Consequently, an optimal choice $n:=N\left(\delta, y^{\delta}\right)$ of the discretization level has to balance between two error terms of different asymptotic behavior: the approximation error that goes to zero and the propagated data noise that in the worst case goes to infinity as $n \rightarrow \infty$. This corresponds to the necessity of correctly choosing a regularization parameter (e.g., $\alpha$ in (3)) in regularization methods for ill-posed problems.

Note that the residual $\left\|\mathbf{T} x_{n}-y\right\|$ can (up to the propagated data noise, whose contribution to the residual is $O(\delta)$ ) be estimated by

$$
\overline{\bar{\gamma}}_{n}:=\left\|\left(I-\mathbf{Q}_{n}\right) \mathbf{T}\right\|
$$

and that, under a source condition (4) the approximation part of the error (i.e., the total error in the noiseless case) goes to zero at some rate

$$
\bar{\gamma}_{n}(\mu):=\left\|\left(I-\mathbf{P}_{n}\right)\left(\mathbf{T}^{*} \mathbf{T}\right)^{\mu}\right\| \leq \overline{\bar{\gamma}}_{n}^{2 \mu},
$$

for $\mu \leq \frac{1}{2}$ (cf., e.g., 21]). Moreover,

$$
\underline{\gamma}_{n} \leq \overline{\bar{\gamma}}_{n-1},
$$

and, in order to obtain optimal convergence rates (5) of $\tilde{x}:=x_{N\left(\delta, y^{\delta}\right)}$ under source conditions (4), it is necessary and sufficient to have, on the other hand, also

$$
\overline{\bar{\gamma}}_{n-1} \leq C_{\underline{\gamma}_{n}}
$$

for some constant $C>0$ (see [22, 17, 18]). Condition (11) says that the data noise amplification factor $1 / \underline{\gamma}_{n}$ (that grows to infinity as $n \rightarrow \infty$ due to the illposedness of (1)) is compensated by a sufficiently good approximation property of the spaces $\mathbf{Y}_{n}$ in combination with the smoothing property of $\mathbf{T}$. It is satisfied, e.g., by truncated SVD, which means that the finite-dimensional spaces $\mathbf{Y}_{n}$ are spanned by eigenvectors of $\mathbf{T T}^{*}$, and which is optimal both with respect to stability and convergence (see, e.g., Section 3.3 in [10], where a more detailed exposition on regularization by projection can be found). For reasons of practical applicability in cases where an explicit SVD is hard or impossible to compute, we here concentrate on projection onto spaces of piecewise polynomial functions, though. In the context of mildly ill-posed first kind integral equations or parameter identification problems 
with spline or finite element discretization $\mathbf{Y}_{n}$, condition (11) seems to be quite natural (cf., e.g., [17], [18]).

In this paper, as in [18], [21], we consider instead of the exact solution $x_{n}$ of (17) its approximation $\tilde{x}_{n}$ by (iterative) multigrid techniques - again with $n:=N\left(\delta, y^{\delta}\right)$ appropriately chosen - as our regularized approximate solution of the infinite-dimensional problem (1).

The asymptotic behavior of the condition of our finite-dimensional system (7) is characterized by the real sequence $\underline{\gamma}_{n}$ : while the largest singular value of $\mathbf{Q}_{n} \mathbf{T} \mathbf{P}_{n}$ is uniformly bounded by the norm of $\underline{T}$, its smallest singular value $\underline{\gamma}_{n}$ goes to zero as $n \rightarrow \infty$. This is due to the ill-posedness of (11) or, in other words, the smoothing property of the forward operator $\mathbf{T}$ that, in combination with the approximation property of the finite-dimensional spaces $\mathbf{Y}_{n}$, makes $\overline{\bar{\gamma}}_{n}$ go to zero as $n \rightarrow \infty$, on the other hand.

To concretize the asymptotics, we denote by $h_{n}$ some discretization parameter (think, e.g., of a mesh size) that geometrically goes to zero as $n \rightarrow \infty$ :

$$
h_{n}=C_{\sigma} \sigma^{n}
$$

for some $\sigma \in(0,1)$, and, by $\mathbf{Y}^{-p}$ a Hilbert space that contains $\mathbf{Y}$ and has a weaker topology:

$$
\mathbf{Y}^{-p} \supset \mathbf{Y}
$$

i.e., we think of $\mathbf{Y}^{-p}$ as a less smooth function space than $\mathbf{Y}$, e.g., $\mathbf{Y}=H^{t}(\Omega)$, $\mathbf{Y}^{-p}=H^{t-p}(\Omega)$, for some $t \in \mathbb{R}, p>0$, and some domain $\Omega$. The real positive number $p$ is supposed to quantify the degree of smoothing by $\mathbf{T}$, which is closely related to the concept of degree of ill-posedness as it is frequently used in the literature on ill-posed problems.

Assumption 1. (Smoothing property of $\mathbf{T}^{*}$ )

$$
\forall v \in \mathbf{Y}: \quad\left\|\mathbf{T}^{*} v\right\|_{\mathbf{X}} \simeq\|v\|_{\mathbf{Y}^{-p}} .
$$

For the pseudo-differential operators of order minus one considered in [5] one has $p=1 / 2$.

In order to be able to exploit this smoothing assumption, we combine it with a condition on the quality of approximation by functions in $\mathbf{Y}_{n}$ :

Assumption 2. (Approximation property of $\mathbf{Y}_{n}$ )

$$
\left\|I-\mathbf{Q}_{n}\right\|_{\mathbf{Y} \rightarrow \mathbf{Y}^{-p}} \leq C h_{n}^{p} .
$$

These assumptions (having in mind Sobolev spaces and piecewise polynomial approximation) yield the following crucial estimate

$$
\overline{\bar{\gamma}}_{n} \leq \overline{\bar{C}} h_{n}^{p},
$$

(and, by (9), $\bar{\gamma}_{n}(\mu)=O\left(h_{n}^{2 p \mu}\right)$ ).

On the other hand, estimating the lowest singular value of the system matrix in (77) corresponds to assuming some kind of inverse inequality on $\mathbf{Y}_{n}$ :

Assumption 3. (Inverse inequality)

$$
\sup _{v \in \mathbf{Y}_{n}} \frac{\|v\|_{\mathbf{Y}}}{\|v\|_{\mathbf{Y}^{-p}}} \leq C h_{n}^{-p}
$$


which, when thinking of the $\mathbf{Y}_{n}$ as piecewise polynomial spaces, seems to be realistic, and from which we get

$$
\underline{\gamma}_{n} \geq \underline{\underline{c}} h_{n}^{p}
$$

(cf. [17). Note that this implies uniform boundedness of the quotient $\overline{\bar{\gamma}}_{n} / \underline{\underline{\gamma}}_{n}$ (i.e., (11)) as well as of the operators $\mathbf{T}\left(\mathbf{Q}_{n} \mathbf{T}\right)^{\dagger} \mathbf{Q}_{n}$ approximating the projection $\mathbf{T} \mathbf{T}^{\dagger}=$ $\left.\operatorname{Proj}_{\mathbf{R}(\mathbf{T})}^{\mathbf{Y}}\right|_{\mathbf{D}\left(\mathbf{T}^{\dagger}\right)}($ cf. $[21])$.

Discrete norms. The discrete inner products used in the respective multigrid approaches below will be based on norm equivalences of the form

$$
\forall v \in \mathbf{Y}_{n}: \quad\|v\|_{\mathbf{Y}^{\prime}}^{2} \simeq \sum_{j=1}^{n} h_{j}^{-2 q}\left\|\left(\mathbf{Q}_{j}-\mathbf{Q}_{j-1}\right) v\right\|_{\mathbf{Y}}^{2}+\left\|\mathbf{Q}_{0} v\right\|_{\mathbf{Y}}^{2},
$$

where $\mathbf{Y}^{\prime}$ is some Hilbert space containing $\mathbf{Y}_{n}, q$ is an index that will be specified below, and the equivalence constants are supposed to be independent of $n$. In the case of $\mathbf{Y}^{\prime}$ being a Sobolev space on a (sufficiently smooth) domain $\Omega, \mathbf{Y}$ being the space of square integrable functions on $\Omega$,

$$
\mathbf{Y}=L^{2}(\Omega), \quad \mathbf{Y}^{\prime}=H^{q}(\Omega),
$$

(as typical, e.g., in parameter identification) and the $\mathbf{Y}_{n}$ being finite element spaces of piecewise polynomial $C^{r}$ functions on a regular and quasi-uniform triangulation, this norm equivalence follows for

$$
-r-3 / 2 \leq q \leq r+3 / 2
$$

from Theorem 15 in Oswald [27].

\section{Two V-CYCLE-CONVERGENT MULTIGRID APPROACHES}

Before proposing concrete multigrid approaches for (11) in subsections 3.1, 3.2, we introduce a general notational framework together with a fundamental convergence assertion, following [6].

Going out from a finite-dimensional variational equation of finding $u \in \mathbf{Y}_{n}$ such that

$$
A(u, v)=f(v) \text { for all } v \in \mathbf{Y}_{n},
$$

where $A(.,$.$) is a positive definite symmetric bilinear form on \mathbf{Y}_{n}$, inducing a norm

$$
\||| u \mid\|=\sqrt{A(u, u)}
$$

and projection operators $P_{k}: \mathbf{Y}_{n} \rightarrow \mathbf{Y}_{k}, k=0, \ldots, n$,

$$
A\left(P_{k} w, v\right)=A(w, v) \quad \text { for all } v \in \mathbf{Y}_{k}
$$

and $f$ a linear functional on $\mathbf{Y}_{n}$, one can, via an additional inner product $\langle.,$.$\rangle on$ $\mathbf{Y}_{n}$ with corresponding projectors $Q_{k}: \mathbf{Y}_{n} \rightarrow \mathbf{Y}_{k}$

$$
\left\langle Q_{k} w, v\right\rangle=\langle w, v\rangle \quad \text { for all } v \in \mathbf{Y}_{k},
$$

and operators $A_{k}: \mathbf{Y}_{k} \rightarrow \mathbf{Y}_{k}$ given by

$$
\left\langle A_{k} w, v\right\rangle=A(w, v) \quad \text { for all } v \in \mathbf{Y}_{k},
$$

(which are spsd. with respect to both inner products $A(.,$.$) and \langle.,$.$\rangle ), and smooth-$ ing operators $S_{k}: \mathbf{Y}_{k} \rightarrow \mathbf{Y}_{k}$, inductively define a symmetric V-cycle multigrid operator $B_{k}^{s}: \mathbf{Y}_{k} \rightarrow \mathbf{Y}_{k}$ by: 
Algorithm 1. (Symmetric MG)

Set $B_{0}^{s}:=\left(A_{0}\right)^{-1}$. Assume that $B_{k-1}^{s}$ has been defined and define $B_{k}^{s} b$ for $b \in \mathbf{Y}_{k}$ as follows:

Set $u:=S_{k} b$.

Set $u:=u+B_{k-1}^{s} Q_{k-1}\left(b-A_{k} u\right)$.

Set $u:=u+S_{k}\left(b-A_{k} u\right)$.

Set $B_{k}^{s} b:=u$.

A nonsymmetric V-cycle MG operator $B_{k}^{n}$ can be defined analogously by omitting the post-smoothing step $u:=u+S_{k}\left(b-A_{k} u\right)$ in Algorithm 1

If, on one hand

$$
S_{k} A_{k} \text { and } I-S_{k} A_{k} \text { are spsd. with respect to } A(., .),
$$

and, on the other hand,

$$
\left\|\mid\left(P_{k}-P_{k-1}\right) v\right\| \| \leq C A\left(S_{k} A_{k} v, v\right) \quad \text { for all } v \in \mathbf{Y}_{k},
$$

Theorem 1 in [6] yields a level independent contraction number estimate, whose proof in our special situation is quite short and will therefore be given explicitly for the convenience of the reader.

Corollary 1 (to Theorem 1 in [6]). Assume that (17) and (18) hold and define $B_{n}^{s}$ and $B_{n}^{n}$ by Algorithm 11 (omitting the post-smoothing step in the second case). Then

(19) $A\left(\left(I-B_{n}^{s} A_{n}\right) u, u\right)=\left\|\mid\left(I-B_{n}^{n} A_{n}\right) u\right\|\left\|^{2} \leq\left(1-\frac{1}{C^{2}}\right)\right\| u\|\|^{2} \quad$ for all $u \in \mathbf{Y}_{n}$.

Remark 1. The constant $C$ in the right-hand side of (19) equals the one in (18), which by (17) must be larger or equal to one.

Proof. It can be easily checked that the sequence of operators $E_{k}: \mathbf{Y}_{n} \rightarrow \mathbf{Y}_{k}$ defined by

$$
\begin{aligned}
& E_{-1}:=I, \\
& E_{k}:=\left(I-B_{k}^{n} A_{k} P_{k}\right)^{*}, \quad k=0,1, \ldots
\end{aligned}
$$

(where the adjoint ${ }^{*}$ is taken w.r.t. $A(.,$.$) ) obeys the recursion$

$$
E_{k}=\left(I-S_{k} A_{k} P_{k}\right) E_{k-1}, \quad k=0,1, \ldots,
$$

where we have set $S_{0}:=A_{0}^{-1}$ for convenience of notation. Therefore, and since by (17) $S_{k} A_{k}-\left(S_{k} A_{k}\right)^{2}$ is spsd., we have, for any $u \in \mathbf{Y}_{n}$,

$$
\begin{aligned}
& \|\| u\left\|\left.\right|^{2}-\right\|\left|\left(I-B_{n}^{n} A_{n}\right)^{*} u \|\right|^{2} \\
& =\left.\sum_{k=0}^{n}\left\|E_{k-1} u\right\|\right|^{2}-\left\|\left|E_{k} u \|\right|^{2}\right. \\
& =\sum_{k=0}^{n}\left(A\left(\left(S_{k} A_{k}-\left(S_{k} A_{k}\right)^{2}\right) P_{k} E_{k-1} u, P_{k} E_{k-1} u\right)\right. \\
& \left.\quad+A\left(S_{k} A_{k} P_{k} E_{k-1} u, P_{k} E_{k-1} u\right)\right) \\
& \geq \sum_{k=0}^{n} A\left(S_{k} A_{k} P_{k} E_{k-1} u, P_{k} E_{k-1} u\right),
\end{aligned}
$$


so that it only remains to show that the latter is greater or equal to $1 / C^{2} \mid\|u\| \|^{2}$. To do so, we decompose

$$
u=\sum_{k=1}^{n}\left(P_{k}-P_{k-1}\right) u+P_{0} u
$$

and get, since $\left(I-E_{k-1}\right) u=\left(B_{k-1}^{n} A_{k-1} P_{k-1}\right)^{*} u \in \mathbf{Y}_{k-1}$ is orthogonal to $\left(P_{k}-P_{k-1}\right) u=\left(I-P_{k-1}\right) P_{k-1} u$, that

$$
\begin{aligned}
& \|u\|\left\|^{2}=\sum_{k=0}^{n} A\left(E_{k-1} u+\left(I-E_{k-1}\right) u,\left(P_{k}-P_{k-1}\right) u\right)+\right\|\left\|P_{0} u\right\| \|^{2} \\
& =\sum_{k=1}^{n} A\left(E_{k-1} u,\left(P_{k}-P_{k-1}\right) u\right)+\|\| P_{0} u\|\|^{2} \\
& \leq \sum_{k=1}^{n}\|\|\left(P_{k}-P_{k-1}\right) E_{k-1} u\|\|\left\|\left|\left(P_{k}-P_{k-1}\right) u\|\|+\left\|\mid P_{0} u\right\|^{2}\right.\right. \\
& \leq \sum_{k=1}^{n} \sqrt{C A\left(S_{k} A_{k} P_{k} E_{k-1} u, P_{k} E_{k-1} u\right)}\|\|\left(P_{k}-P_{k-1}\right) u\|\| \\
& +\sqrt{A\left(S_{0} A_{0} P_{0} E_{-1} u, P_{0} E_{-1} u\right)}\left\|\left|P_{0} u \|\right|\right. \\
& \leq \sqrt{\max \{C, 1\} \sum_{k=0}^{n} A\left(S_{k} A_{k} P_{k} E_{k-1} u, P_{k} E_{k-1} u\right)}\|u\| \|
\end{aligned}
$$

where we have used (18) with $v:=P_{k} E_{k-1} u$ and the fact that we have set $S_{0} A_{0} P_{0}=$ $P_{0}$ in the fourth line, and Cauchy-Schwarz in $\mathbb{R}^{n}$ yielded the last inequality.

In our situation, the bilinear form $A$ and the projections $P_{k}$ are, due to (8)), given by

$$
A(w, v):=\left\langle\mathbf{T}^{*} w, \mathbf{T}^{*} v\right\rangle_{\mathbf{X}}, \quad P_{k}:=\left(\left.\mathbf{T}^{*}\right|_{\mathbf{Y}_{k} \rightarrow \mathbf{X}_{k}}\right)^{-1} \mathbf{P}_{k} \mathbf{T}^{*}
$$

(where $\left(\left.\mathbf{T}^{*}\right|_{\mathbf{Y}_{k} \rightarrow \mathbf{X}_{k}}\right)^{-1}$ has to be understood as the inverse of the bijective restriction $\mathbf{T}^{*}: \mathbf{Y}_{k} \rightarrow \mathbf{X}_{k}$ of $\mathbf{T}^{*}$ to the finite-dimensional spaces on the $k$-th level) and the right-hand side by

$$
f=\left\langle y^{\delta}, .\right\rangle \mathbf{Y}
$$

The behavior of the multigrid method obviously heavily depends on the choice of the smoothers $S_{k}$ and the inner products $\langle.,$.$\rangle . In the following we describe$ two approaches that will lead to level independent contraction factor estimates according to Corollary [1]

3.1. Generalization of Bramble, Leyk, Pasciak (1994). The ill-posedness and its complicating consequences in the context of MGM are reflected in the fact that the (generalized) inverse of $\mathbf{T}^{*}$ is unbounded on its domain with respect to the topologies of $\mathbf{X}$ and $\mathbf{Y}$. The norm of the auxiliary space $\mathbf{Y}^{-p}$ in Assumption 1 is just weak enough so that the (generalized) inverse of $\mathbf{T}^{*}$ as a mapping from its domain in $\mathbf{X}$ to $\mathbf{Y}^{-p}$ is bounded. Now, let $\mathbf{Y}^{-2 p}$ denote a Hilbert space containing $\mathbf{Y}, \mathbf{Y}^{-p}$, with still weaker norm:

$$
\mathbf{Y}^{-2 p} \supset \mathbf{Y}^{-p} \supset \mathbf{Y} .
$$


Then $\mathbf{T}^{*}$ acts on (its domain within) $\mathbf{Y}^{-2 p}$ like a differential operator, i.e., its inverse is smoothing. Hence, with respect to the topology of $\mathbf{Y}^{-2 p}$, we are in a situation similar to well-posed positive order differential equations, where the construction of smoothers for MGM is well understood. By means of discrete norms, the resulting operators can be "lifted" to the original topology. Based on this idea, in [3] a smoother is constructed for the case $\mathbf{Y}=L^{2}(\Omega), \mathbf{Y}^{-2 p}=H^{-1}(\Omega)(\Omega$ some regular domain) and is shown to yield a V-cycle convergent MGM for pseudo-differential operators of order -1 . This construction as well as the convergence proof can be carried over to general ill-posed equations (1) as follows.

Returning to the notation of above, here, $\langle.,$.$\rangle is chosen to induce a norm weaker$ than $\||| \cdot||$, namely, in our context,

$$
\langle w, v\rangle:=\left\langle\mathbf{T} \mathbf{T}^{*} w, \mathbf{T T}^{*} v\right\rangle_{\mathbf{Y}} .
$$

Its discrete implementation $\langle., .\rangle_{k}$ is based on the norm equivalence (15) with $\mathbf{Y}^{\prime}=$ $\mathbf{Y}^{-p}, q=-p$, i.e.,

$$
\forall v \in \mathbf{Y}_{n}: \quad\|v\|_{\mathbf{Y}^{-p}}^{2} \simeq \sum_{j=1}^{n} h_{j}^{2 p}\left\|\left(\mathbf{Q}_{j}-\mathbf{Q}_{j-1}\right) v\right\|_{\mathbf{Y}}^{2}+\left\|\mathbf{Q}_{0} v\right\|_{\mathbf{Y}}^{2},
$$

and the additional assumptions

$$
\begin{gathered}
\forall v \in \mathbf{Y}: \quad\left\|\mathbf{T T}^{*} v\right\|_{\mathbf{Y}} \geq c\|v\|_{\mathbf{Y}^{-2 p}}, \\
\forall v \in \mathbf{Y}_{k}: \quad\|v\|_{\mathbf{Y}^{-2 p}}^{2} \geq c\left(\sum_{j=1}^{k} h_{j}^{4 p}\left\|\left(\mathbf{Q}_{j}-\mathbf{Q}_{j-1}\right) v\right\|_{\mathbf{Y}}^{2}+\left\|\mathbf{Q}_{0} v\right\|_{\mathbf{Y}}^{2}\right),
\end{gathered}
$$

where $\mathbf{Y}^{-2 p}$ is, as indicated by the superscript, a function space with weaker topol-

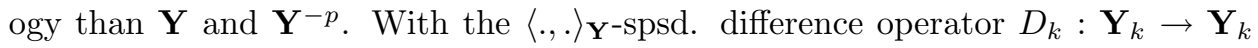
defined by

$$
D_{k} v=\sum_{j=1}^{k} h_{j}^{-4 p}\left(\mathbf{Q}_{j}-\mathbf{Q}_{j-1}\right) v+\mathbf{Q}_{0} v
$$

and the discrete inner product $\langle., .\rangle_{k}$ given by

$$
\langle v, w\rangle_{k}=\left\langle D_{k}^{-1} v, w\right\rangle_{\mathbf{Y}},
$$

we get, with some constant $C_{-}$independent of $k$ :

$$
\forall v \in \mathbf{Y}_{k}: \quad\|v\|_{k} \leq C_{-}\left\|\mathbf{T} \mathbf{T}^{*} v\right\|_{\mathbf{Y}} ;
$$

moreover, by (22),

$$
\forall v \in \mathbf{Y}_{k}: \quad\|v\|_{\mathbf{Y}^{-p}} \simeq\left\|D_{k}^{-1 / 4} v\right\|_{\mathbf{Y}} .
$$

Following [3], we define the smoothing operator $S_{k}: \mathbf{Y}_{k} \rightarrow \mathbf{Y}_{k}$ by

$$
\left\langle S_{k} w, v\right\rangle_{k}=\frac{1}{\xi_{k}}\langle w, v\rangle \quad \text { for all } v \in \mathbf{Y}_{k},
$$

where

$$
C \sup _{v \in \mathbf{Y}_{k}} \frac{\|v\| \|^{2}}{\|v\|_{k}^{2}} \geq \xi_{k} \geq \sup _{v \in \mathbf{Y}_{k}} \frac{\|\| v\|\|^{2}}{\|v\|_{k}^{2}},
$$

for some constant $C>0$, i.e., $\xi_{k} \sim h_{k}^{-2 p}$,

$$
S_{k}=\frac{1}{\xi_{k}} D_{k} \mathbf{Q}_{k}\left(\mathbf{T T}^{*}\right)^{2}
$$


so that

$$
S_{k} A_{k}=\frac{1}{\xi_{k}} D_{k} \mathbf{Q}_{k} \mathbf{T T}^{*}
$$

is symmetric and (17) holds by

$$
\begin{aligned}
& A\left(S_{k} A_{k} v, v\right)=\frac{1}{\xi_{k}}\left\langle D_{k} \mathbf{Q}_{k} \mathbf{T} \mathbf{T}^{*} v, \mathbf{T} \mathbf{T}^{*} v\right\rangle_{\mathbf{Y}}=\frac{1}{\xi_{k}}\left\|D_{k} \mathbf{Q}_{k} \mathbf{T} \mathbf{T}^{*} v\right\|_{k}^{2} \geq 0, \\
& A\left(S_{k} A_{k} v, v\right) \leq \frac{1}{\xi_{k}}\left\|D_{k} \mathbf{Q}_{k} \mathbf{T} \mathbf{T}^{*} v\right\|\|\|\|v\| \mid \\
& \leq \frac{1}{\sqrt{\xi_{k}}}\left\|D_{k} \mathbf{Q}_{k} \mathbf{T T}^{*} v\right\|_{k}\|\| v\|\| \\
& =\sqrt{A\left(S_{k} A_{k} v, v\right)}\||v|\|,
\end{aligned}
$$

where we have used the definition of $\xi_{k}$ according to (27). On the other hand, the upper bound on $\xi_{k}$ in (27) implies, by the identity $\mathbf{Q}_{n} \mathbf{T}\left[I-\mathbf{P}_{n}\right]=0$, (13), Assumption 1 and (26):

$$
\begin{aligned}
\xi_{k}\left\|\mathbf{T}\left(I-\mathbf{P}_{k-1}\right)\right\|^{2} & \leq \xi_{k} \overline{\bar{\gamma}}_{k-1}^{2} \\
& \leq C h_{k}^{2 p}\left(\sup _{v \in \mathbf{Y}_{k}} \frac{\left\|D_{k}^{-1 / 4} v\right\|_{\mathbf{Y}}}{\left\|D_{k}^{-1 / 2} v\right\|_{\mathbf{Y}}}\right)^{2} \\
& =C h_{k}^{2 p}\left\|D_{k}^{1 / 4}\right\|_{\mathbf{Y} \rightarrow \mathbf{Y}}^{2} \leq C_{\xi \overline{\bar{\gamma}}}
\end{aligned}
$$

for some constant $C_{\xi \overline{\bar{\gamma}}}>0$, so that, by Cauchy-Schwarz, (21), and (25), we get

$$
\begin{aligned}
A\left(S_{k} A_{k} v, v\right) & =\frac{1}{\xi_{k}}\left\|D_{k}^{1 / 2} \mathbf{Q}_{k} \mathbf{T T}^{*} v\right\|_{\mathbf{Y}}^{2} \\
& \geq \frac{1}{\xi_{k}}\left(\frac{\left\langle D_{k}^{1 / 2} \mathbf{Q}_{k} \mathbf{T} \mathbf{T}^{*} v, D_{k}^{-1 / 2}\left(P_{k}-P_{k-1}\right) v\right\rangle_{\mathbf{Y}}}{\left\|D_{k}^{-1 / 2}\left(P_{k}-P_{k-1}\right) v\right\|_{\mathbf{Y}}}\right)^{2} \\
& =\frac{1}{\xi_{k}}\left(\frac{\left\|\left(P_{k}-P_{k-1}\right) v\right\| \|^{2}}{\left\|\left(P_{k}-P_{k-1}\right) v\right\|_{k}}\right)^{2} \\
& \geq \frac{1}{C_{-} \xi_{k}}\left(\frac{\left\|\left(I-\mathbf{P}_{k-1}\right) \mathbf{P}_{k} \mathbf{T}^{*} v\right\|_{\mathbf{X}}}{\left\|\mathbf{T}\left(I-\mathbf{P}_{k-1}\right) \mathbf{P}_{k} \mathbf{T}^{*} v\right\|_{\mathbf{Y}}}\right)^{2}\left\|\left(P_{k}-P_{k-1}\right) v\right\| \|^{2} \\
& \geq \frac{1}{C_{-} C_{\xi \bar{\gamma}}}\left\|\left(P_{k}-P_{k-1}\right) v\right\| \|^{2} .
\end{aligned}
$$

Taking into account the fact that here

$$
\begin{aligned}
Q_{k} & =\left(\mathbf{Q}_{k}\left(\mathbf{T T}^{*}\right)^{2} \mathbf{Q}_{k}\right)^{-1} \mathbf{Q}_{k}\left(\mathbf{T T}^{*}\right)^{2}, \\
A_{k} & =\left(\mathbf{Q}_{k}\left(\mathbf{T T}^{*}\right)^{2} \mathbf{Q}_{k}\right)^{-1} \mathbf{Q}_{k} \mathbf{T} \mathbf{T}^{*} \mathbf{Q}_{k}
\end{aligned}
$$

and setting

$$
\mathbf{S}_{k}:=\frac{1}{\xi_{k}} \mathbf{T}^{*} D_{k},
$$

we can transform Algorithm 1 (or its nonsymmetric version) to define a multigrid operator $\mathbf{B}_{n}^{s}\left(\right.$ or $\mathbf{B}_{n}^{n}$ ) for the iterative solution or preconditioning of equation (7)): 
Algorithm 2. Set $\mathbf{B}_{0}^{s}:=\left(\mathbf{Q}_{0} \mathbf{T} \mathbf{P}_{0}\right)^{-1}$. Assume that $\mathbf{B}_{k-1}^{s}$ has been defined and define $\mathbf{B}_{k}^{s} b$ for $b \in \mathbf{Y}_{k}$ as follows:

Set $x:=\mathbf{S}_{k} b$.

Set $x:=x+\mathbf{B}_{k-1}^{s} \mathbf{Q}_{k-1}\left(b-\mathbf{Q}_{k} \mathbf{T} x\right)$.

Set $x:=x+\mathbf{S}_{k}\left(b-\mathbf{Q}_{k} \mathbf{T} x\right)$.

Set $\mathbf{B}_{k}^{s} b:=x$. 2

The nonsymmetric version $\mathbf{B}_{k}^{n}$ is defined by omitting the fifth line of Algorithm

It can be easily seen by induction that the so-defined $\mathbf{B}_{k}^{s}\left(\mathbf{B}_{k}^{n}\right)$ is related to $B_{k}^{s}$ $\left(B_{k}^{n}\right)$ by

and hence we have

$$
\mathbf{B}_{k}^{s / n}=\mathbf{T}^{*} B_{k}^{s / n}\left(\mathbf{Q}_{k}\left(\mathbf{T T}^{*}\right)^{2} \mathbf{Q}_{k}\right)^{-1}
$$

$$
\mathbf{B}_{k}^{s / n} \mathbf{Q}_{k} \mathbf{T P}_{k}=\mathbf{T}^{*} B_{k}^{s / n} A_{k}
$$

Corollary 1 together with (29), (30), (31), (32) therefore implies

Corollary 2. Let Assumptions 1, 2, 3, and (22), (23), (24) hold and define $\mathbf{B}_{n}^{s}$ and $\mathbf{B}_{n}^{n}$ by Algorithm $\mathbf{Q}$ with $\mathbf{S}_{k}$ according to (28). Then

$$
\left\langle\left(I-\mathbf{B}_{n}^{s} \mathbf{Q}_{n} \mathbf{T}\right) x, x\right\rangle_{\mathbf{X}}=\left\|\left(I-\mathbf{B}_{n}^{n} \mathbf{Q}_{n} \mathbf{T}\right) x\right\|_{\mathbf{X}}^{2} \leq c\|x\|_{\mathbf{X}}^{2} \quad \text { for all } x \in \mathbf{X}_{n}
$$

for some $c<1$ independent of $n$.

The contraction factor result (34) is given here in the form in which it is needed for the theory yielding the regularization result, Corollary 5 below. For a practical implementation one will of course not work in $\mathbf{X}_{k}$ but in $\mathbf{Y}_{k}$, where one usually has a convenient basis available, approximating a solution to the system in the second line of (8) and subsequently applying $\mathbf{T}^{*}$, as prescribed by the first line of (8). For this purpose, we also give here the matrix form of Algorithm 2 with (28) and of Corollary 2. With $b^{k} \in \mathbb{R}^{\operatorname{dim}\left(\mathbf{Y}_{k}\right)}$ being the vector of coefficients of $b \in \mathbf{Y}_{k}$ with respect to some basis $\left\{\psi_{1}^{k}, \ldots, \psi_{\operatorname{dim}\left(\mathbf{Y}_{k}\right)}^{k}\right\}$ of $\mathbf{Y}_{k}$,

$$
b=\sum_{j=1}^{\operatorname{dim}\left(\mathbf{Y}_{k}\right)} b_{j}^{k} \psi_{j}^{k}
$$

and with the matrices

$$
\begin{aligned}
\bar{D}^{k} & :=\left(\left\langle D_{k} \psi_{i}^{k}, \psi_{j}^{k}\right\rangle_{\mathbf{Y}}\right)_{1 \leq i, j \leq \operatorname{dim}\left(\mathbf{Y}_{k}\right)}, \\
\bar{G}^{k} & :=\left(\left\langle\psi_{i}^{k}, \psi_{j}^{k}\right\rangle_{\mathbf{X}}\right)_{1 \leq i, j \leq \operatorname{dim}\left(\mathbf{Y}_{k}\right)}, \\
\bar{M}^{k} & :=\left(\left\langle\mathbf{T}^{*} \psi_{i}^{k}, \mathbf{T}^{*} \psi_{j}^{k}\right\rangle_{\mathbf{X}}\right)_{1 \leq i, j \leq \operatorname{dim}\left(\mathbf{Y}_{k}\right)},
\end{aligned}
$$

and $\bar{C}^{k-1} \in \mathbb{R}_{\operatorname{dim}\left(\mathbf{Y}_{k-1}\right)}^{\operatorname{dim}\left(\mathbf{Y}_{k}\right)}$ such that, by (6)

$$
\left(\psi_{1}^{k-1}, \ldots, \psi_{\operatorname{dim}\left(\mathbf{Y}_{k-1}\right)}^{k-1}\right)^{T}=\bar{C}^{k-1}\left(\psi_{1}^{k}, \ldots, \psi_{\operatorname{dim}\left(\mathbf{Y}_{k}\right)}^{k}\right)^{T}
$$

we can write

$$
\mathbf{B}_{k}^{s / n} b=\mathbf{T}^{*} \sum_{j=1}^{\operatorname{dim}\left(\mathbf{Y}_{k}\right)}\left({\overline{\mathbf{B}^{s / n}}}^{k} b^{k}\right)_{j} \psi_{j}^{k}
$$


where

Algorithm 3. (Matrix form of Algorithm 2 with (28))

Set $\overline{\mathbf{B}}^{0}:=\left(\bar{M}^{0}\right)^{-1} \bar{G}^{0}$. Assume that $\overline{\mathbf{B}}^{k-1}$ has been defined and define $\overline{\mathbf{B}}^{k} b^{k}$ for $b^{k} \in \mathbb{R}^{\operatorname{dim}\left(\mathbf{Y}_{k}\right)}$ as follows:

Set $x^{k}:=\frac{1}{\xi_{k}}\left(\bar{G}^{k}\right)^{-1} \bar{D}^{k} b^{k}$.

Set $x^{k}:=x^{k}+\left(\bar{C}^{k-1}\right)^{T} \overline{\mathbf{B}}^{k-1} \bar{G}^{k-1} \bar{C}^{k-1}\left(\bar{G}^{k} b^{k}-\bar{M}^{k} x^{k}\right)$.

Set $x^{k}:=x^{k}+\frac{1}{\xi_{k}}\left(\bar{G}^{k}\right)^{-1} \bar{D}^{k}\left(b^{k}-\left(\bar{G}^{k}\right)^{-1} \bar{M}^{k} x^{k}\right)$.

Set $\overline{\mathbf{B}}^{k} b^{k}:=x^{k}$.

(To get $\overline{\mathbf{B}}^{k}$, omit the fifth line in Algorithm[3.)

The result (34) of Corollary 2 in matrix form reads as

$$
\begin{array}{r}
\left\langle\left(\bar{I}^{n}-{\overline{\mathbf{B}^{s}}}^{n}\left(\bar{G}^{n}\right)^{-1} \bar{M}^{n}\right) x^{n}, x^{n}\right\rangle_{\bar{M}^{n}}=\left\|\left(\bar{I}^{n}-{\overline{\mathbf{B}^{n}}}^{n}\left(\bar{G}^{n}\right)^{-1} \bar{M}^{n}\right) x^{n}\right\|_{\bar{M}^{n}}^{2} \leq c\left\|x^{n}\right\|_{\bar{M}^{n}}^{2} \\
\text { for all } x^{n} \in \mathbb{R}^{\operatorname{dim}\left(\mathbf{Y}_{n}\right)},
\end{array}
$$

where $\bar{I}^{n}$ is the $\operatorname{dim}\left(\mathbf{Y}_{n}\right)$-dimensional identity matrix and $\langle\cdot, \cdot\rangle_{\bar{M}^{n}}$ and $\|\cdot\|_{\bar{M}^{n}}$ are the energy inner product and norm, respectively, with respect to the system matrix $\bar{M}^{n}:\left\langle x_{1}^{n}, x_{2}^{n}\right\rangle_{\bar{M}^{n}}:=\left(x_{1}^{n}\right)^{T} \bar{M}^{n} x_{2}^{n},\left\|x_{1}^{n}\right\|_{\bar{M}^{n}}:=\sqrt{\left\langle x_{1}^{n}, x_{1}^{n}\right\rangle_{\bar{M}^{n}}}, x_{1}^{n}, x_{2}^{n} \in \mathbb{R}^{\operatorname{dim}\left(\mathbf{Y}_{n}\right)}$.

3.2. Modification of King (1992). The smoother proposed in 22] in our context reads as

$$
S_{k}:=c_{\xi} h_{k}^{-2 p}\left(I-\mathbf{Q}_{k-1}\right) \mathbf{Q}_{k} .
$$

By the projection $\mathbf{Q}_{k-1}$, it separates $\mathbf{Y}_{k}$ into a relatively low and a relatively high frequency part. On the high frequencies it acts as a very simple approximation of the inverse of $\mathbf{Q}_{k} \mathbf{T} \mathbf{T}^{*} \mathbf{Q}_{k}$ (note that by Assumptions 2, 3, and (12), the eigenvalues of $\left(I-\mathbf{Q}_{k-1}\right) \mathbf{Q}_{k} \mathbf{T} \mathbf{T}^{*} \mathbf{Q}_{k}\left(I-\mathbf{Q}_{k-1}\right)$ are proportional to $\left.h_{k}^{2 p}\right)$, while just removing the low frequencies. It is intuitively clear that it could be improved by replacing the removal of the low frequency part by a more refined treatment, e.g., by a perpetuation of the principle described above, to the lower levels:

$$
S_{k}:=c_{\xi} \sum_{j=1}^{k} h_{j}^{-2 p}\left(I-\mathbf{Q}_{j-1}\right) \mathbf{Q}_{j}+\mathbf{Q}_{0} .
$$

In the framework of the general Corollary 1 , this means that we choose $\langle.,$.$\rangle to$ be equal to the $\mathbf{Y}$-inner product, i.e.,

$$
\langle., .\rangle=\langle., .\rangle_{\mathbf{Y}},
$$

hence this time corresponding to a stronger norm than \|\|$.\|\|$ on $\mathbf{Y}_{k}$. The norm equivalence (15), which we assume here to hold for $\mathbf{Y}^{\prime}=\left(\mathbf{Y}^{-p}\right)^{*}, q=p$, i.e.,

$$
\forall v \in \mathbf{Y}_{n}: \quad\|v\|_{\left(\mathbf{Y}^{-p}\right)^{*}}^{2} \simeq \sum_{j=1}^{n} h_{j}^{-2 p}\left\|\left(\mathbf{Q}_{j}-\mathbf{Q}_{j-1}\right) v\right\|_{\mathbf{Y}}^{2}+\left\|\mathbf{Q}_{0} v\right\|_{\mathbf{Y}}^{2}
$$

in combination with Assumption 1 is used here to analyze the smoothing operator $S_{k}$, that can be rewritten as

$$
S_{k}=c_{\xi} \hat{D}_{k}
$$


where the difference operator $\hat{D}_{k}: \mathbf{Y}_{k} \rightarrow \mathbf{Y}_{k}$ is the square root of $D_{k}$ from subsection 3.1

$$
\hat{D}_{k} v=\sum_{j=1}^{k} h_{j}^{-2 p}\left(\mathbf{Q}_{j}-\mathbf{Q}_{j-1}\right) v+\mathbf{Q}_{0} v,
$$

so that, by our assumptions,

$$
c_{\hat{D}}\|v\|_{\left(\mathbf{Y}^{-p}\right)^{*}} \leq\left\langle\hat{D}_{k} v, v\right\rangle_{\mathbf{Y}} \leq C_{\hat{D}}\|v\|_{\left(\mathbf{Y}^{-p}\right)^{*}},
$$

and the constant $c_{\xi}$ is chosen such that

$$
0<c_{\xi} \leq \frac{1}{C_{\hat{D}}\left\|\mathbf{T}^{*}\right\|_{\mathbf{Y}^{-p} \rightarrow \mathbf{X}}} .
$$

The operator $S_{k} A_{k}$ is then clearly symmetric with respect to $A(.,$.$) and$

$$
\begin{aligned}
0 \leq A\left(S_{k} A_{k} v, v\right) & =c_{\xi}\left(\sum_{j=1}^{k} h_{j}^{-2 p}\left\|\left(\mathbf{Q}_{j}-\mathbf{Q}_{j-1}\right) A_{k} v\right\|_{\mathbf{Y}}^{2}+\left\|\mathbf{Q}_{0} A_{k} v\right\|_{\mathbf{Y}}^{2}\right) \\
& \leq c_{\xi} C_{\hat{D}}\left\|A_{k} v\right\|_{\left(\mathbf{Y}^{-p}\right)^{*}}^{2} \\
& =c_{\xi} C_{\hat{D}}\left(\sup _{w \in \mathbf{Y}_{k}} \frac{\left\langle\mathbf{T}^{*} v, \mathbf{T}^{*} w\right\rangle_{\mathbf{X}}}{\|w\|_{\mathbf{Y}}^{-p}}\right)^{2} \\
& \leq c_{\xi} C_{\hat{D}}\left\|\mathbf{T}^{*}\right\|_{\mathbf{Y}^{-p} \rightarrow \mathbf{X}}\|v\| \|^{2} .
\end{aligned}
$$

Analogously, one sees that

$$
A\left(S_{k} A_{k} v, v\right) \geq c \mid\|v\| \|^{2}
$$

for some $c>0$.

By the fact that now

$$
Q_{k}=\mathbf{Q}_{k}, \quad A_{k}=\mathbf{Q}_{k} \mathbf{T} \mathbf{T}^{*} \mathbf{Q}_{k},
$$

we obtain, as in the section above, multigrid operators $\mathbf{B}_{n}^{s}, \mathbf{B}_{n}^{n}$ for (7) from Algorithm 2, this time setting

$$
\mathbf{S}_{k}:=c_{\xi} \mathbf{T}^{*} \hat{D}_{k}
$$

Note that the so-defined $\mathbf{B}_{k}^{s}, \mathbf{B}_{k}^{n}$ satisfy

$$
\mathbf{B}_{k}^{s / n}=\mathbf{T}^{*} B_{k}^{s / n},
$$

as well as (33), so that Corollary 1 together with (42), (43) implies

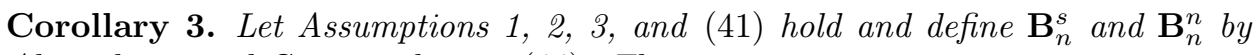
Algorithm 2 with $\mathbf{S}_{k}$ according to (44). Then

$$
\left\langle\left(I-\mathbf{B}_{n}^{s} \mathbf{Q}_{n} \mathbf{T}\right) x, x\right\rangle_{\mathbf{X}}=\left\|\left(I-\mathbf{B}_{n}^{n} \mathbf{Q}_{n} \mathbf{T}\right) x\right\|_{\mathbf{X}}^{2} \leq c\|x\|_{\mathbf{X}}^{2} \quad \text { for all } x \in \mathbf{X}_{n}
$$

for some $c<1$ independent of $n$.

In matrix notation with respect to a basis of $\mathbf{Y}_{k}$ and using the notations 35(39) of the previous subsection, Algorithm 2 with (44) by (35), (36), (37), (38), (39) and

$$
\hat{\bar{D}}^{k}:=\left(\left\langle\hat{D}_{k} \psi_{i}^{k}, \psi_{j}^{k}\right\rangle_{\mathbf{Y}}\right)_{1 \leq i, j \leq \operatorname{dim}\left(\mathbf{Y}_{k}\right)}
$$


becomes

Algorithm 4. (Matrix form of Algorithm 2 with (44))

Set $\overline{\mathbf{B}}^{0}:=\left(\bar{M}^{0}\right)^{-1} \bar{G}^{0}$. Assume that $\overline{\mathbf{B}}^{k-1}$ has been defined and define $\overline{\mathbf{B}}^{k} b^{k}$ for $b^{k} \in \mathbb{R}^{\operatorname{dim}\left(\mathbf{Y}_{k}\right)}$ as follows:

Set $x^{k}:=c_{\xi}\left(\bar{G}^{k}\right)^{-1} \hat{\bar{D}}^{k} b^{k}$.

Set $x^{k}:=x^{k}+\left(\bar{C}^{k-1}\right)^{T} \overline{\mathbf{B}}^{k-1} \bar{G}^{k-1} \bar{C}^{k-1}\left(\bar{G}^{k} b^{k}-\bar{M}^{k} x^{k}\right)$.

Set $x^{k}:=x^{k}+c_{\xi}\left(\bar{G}^{k}\right)^{-1} \hat{\bar{D}}^{k}\left(b^{k}-\left(\bar{G}^{k}\right)^{-1} \bar{M}^{k} x^{k}\right)$.

Set $\overline{\mathbf{B}}^{k} b^{k}:=x^{k}$.

(To get $\overline{\mathbf{B}}^{k}$, omit the fifth line in Algorithm 4.)

As in the previous subsection, the result (45) of Corollary 3 can be rewritten in matrix form as (40).

Since it is just a straightforward consequence of the norm equivalence assumptions made (cf. inequalities (42) and (43)), we finally also mention the uniform preconditioning property of the additive $B P X$ preconditioner from [7]

$$
\mathbf{B}_{n}^{a}=c_{\xi} \mathbf{T}^{*}\left(\sum_{k=1}^{n} h_{k}^{-2 p}\left(\mathbf{Q}_{k}-\mathbf{Q}_{k-1}\right)+\mathbf{Q}_{0}\right):
$$

Corollary 4. Let Assumptions 1, 2, 3, and (41) hold and define $\mathbf{B}_{n}^{a}$ by (46). Then

$$
0 \leq\left\langle\left(I-\mathbf{B}_{n}^{a} \mathbf{Q}_{n} \mathbf{T} \mathbf{P}_{n}\right) x, x\right\rangle_{\mathbf{X}} \leq c\|x\|_{\mathbf{X}}^{2} \quad \text { for all } x \in \mathbf{X}_{n}
$$

for some $c<1$ independent of $n$.

Remark 2. In applications like parameter identification, the evaluation of $\mathbf{T}$ (and of $\mathbf{T}^{*}$ ) will often involve the solution of a PDE, which usually has to be done in an approximate way. In that case it will be more appropriate not to explicitly calculate the entries of the matrix $\bar{M}^{k}$, but to assemble $u_{k}:=\sum_{j=1}^{\operatorname{dim}\left(\mathbf{Y}_{k}\right)} x_{j}^{k} \psi_{j}^{k}$ and (approximately) compute the coefficients of $\mathbf{Q}_{n} \mathbf{T} \mathbf{T}^{*} u_{k}$, in the coarse grid correction (and in the post-smoothing step) of the respective multigrid algorithm. To derive sufficient closeness conditions for the numerical approximations $\tilde{\mathbf{T}}_{k}, \tilde{\mathbf{T}}_{k}^{*}$ of $\mathbf{T}, \mathbf{T}^{*}$ on each level $k$ (where $\tilde{\mathbf{T}}_{k}^{*}$ need not necessarily be the adjoint of $\tilde{\mathbf{T}}_{k}$ ), we denote by a tilde the respective perturbed operators produced by Algorithm 2 when using $\tilde{\mathbf{T}}_{k}, \tilde{\mathbf{T}}_{k}^{*}$ instead of $\mathbf{T}, \mathbf{T}^{*}$ on the $k$-th level. It is straightforward to see that the difference between the preconditioned (unperturbed) operator on the $k$-th level with perturbed and with unperturbed nonsymmetric preconditioner, respectively, obeys the recursion

$$
\begin{aligned}
\tilde{\mathbf{B}}_{k}^{n} \mathbf{Q}_{k} \mathbf{T}-\mathbf{B}_{k}^{n} \mathbf{Q}_{k} \mathbf{T}= & \left(\tilde{\mathbf{B}}_{k-1}^{n} \mathbf{Q}_{k-1} \mathbf{T}-\mathbf{B}_{k-1}^{n} \mathbf{Q}_{k-1} \mathbf{T}\right)\left(I-\tilde{\mathbf{S}}_{k} \mathbf{Q}_{k} \mathbf{T}\right) \\
& +\left(I-\mathbf{B}_{k-1}^{n} \mathbf{Q}_{k-1} \mathbf{T}\right)\left(\tilde{\mathbf{S}}_{k} \mathbf{Q}_{k} \mathbf{T}-\mathbf{S}_{k} \mathbf{Q}_{k} \mathbf{T}\right) \\
& -\tilde{\mathbf{B}}_{k-1}^{n} \mathbf{Q}_{k-1}\left(\tilde{\mathbf{T}}_{k}-\mathbf{T}\right) \tilde{\mathbf{S}}_{k} \mathbf{Q}_{k} \mathbf{T}
\end{aligned}
$$

for all $k \leq n$, so that for the real sequences

$$
\begin{gathered}
\epsilon_{k}:=\left\|\tilde{\mathbf{B}}_{k}^{n} \mathbf{Q}_{k} \mathbf{T}-\mathbf{B}_{k}^{n} \mathbf{Q}_{k} \mathbf{T}\right\| \mathbf{X} \rightarrow \mathbf{X}, \\
\alpha_{k}:=\left\|\tilde{\mathbf{S}}_{k} \mathbf{Q}_{k} \mathbf{T}-\mathbf{S}_{k} \mathbf{Q}_{k} \mathbf{T}\right\| \mathbf{X} \rightarrow \mathbf{X}, \quad \beta_{k}:=\left\|\mathbf{Q}_{k-1}\left(\tilde{\mathbf{T}}_{k}-\mathbf{T}\right)\right\| \mathbf{X} \rightarrow \mathbf{Y}
\end{gathered}
$$


we get

$$
\begin{aligned}
\epsilon_{k} \leq & \epsilon_{k-1}\left(2\left(1+\frac{\overline{\bar{c}}}{\underline{\underline{c}}}\right)+\alpha_{k}\right)\left(1+\frac{\beta_{k}}{\underline{\gamma}_{k-1}}\right) \\
& +(1+c)\left(1+\frac{\overline{\bar{C}}}{\underline{\underline{c}}}\right) \alpha_{k}+(1+c)\left(1+\frac{\overline{\bar{C}}}{\underline{\underline{c}}}+\alpha_{k}\right) \frac{\beta_{k}}{\underline{\gamma}_{k-1}},
\end{aligned}
$$

where we have used the estimates

$$
\begin{aligned}
\|(I- & \left.\mathbf{B}_{k-1}^{n} \mathbf{Q}_{k-1} \mathbf{T}\right) \| \mathbf{x} \rightarrow \mathbf{x} \\
= & \|\left(I-\mathbf{B}_{k-1}^{n} \mathbf{Q}_{k-1} \mathbf{T}\right) \mathbf{P}_{k-1}+\left(I-\mathbf{P}_{k-1}\right) \\
& -\mathbf{B}_{k-1}^{n} \mathbf{Q}_{k-1} \mathbf{T}\left(\mathbf{Q}_{k-1} \mathbf{T}\right)^{\dagger} \mathbf{Q}_{k-1} \mathbf{T}\left(I-\mathbf{P}_{k-1}\right) \|_{\mathbf{X} \rightarrow \mathbf{X}} \\
= & \| \underbrace{\left(I-\mathbf{B}_{k-1}^{n} \mathbf{Q}_{k-1} \mathbf{T}\right) \mathbf{P}_{k-1}}_{\|\cdot\| \mathbf{x} \rightarrow \mathbf{x} \leq c}+\left(I-\mathbf{P}_{k-1}\right) \\
& -\underbrace{\mathbf{B}_{k-1}^{n} \mathbf{Q}_{k-1} \mathbf{T} \mathbf{P}_{k-1}}_{\|\cdot\| \mathbf{x} \rightarrow \mathbf{x} \leq 1+c} \underbrace{\left(\mathbf{Q}_{k-1} \mathbf{T}\right)^{\dagger} \mathbf{Q}_{k-1}}_{\|\cdot\| \mathbf{Y} \rightarrow \mathbf{x}=1 / \underline{\gamma}_{k-1}} \underbrace{\mathbf{T}\left(I-\mathbf{P}_{k-1}\right)}_{\|\cdot\| \mathbf{x} \rightarrow \mathbf{Y} \leq \overline{\bar{\gamma}}_{k-1}} \|_{\mathbf{X} \rightarrow \mathbf{X}} \\
\leq & (1+c)\left(1+\frac{\overline{\bar{c}}}{\underline{c}}\right)
\end{aligned}
$$

(cf. (13), (14), (34), (45)),

$$
\begin{gathered}
\left\|\tilde{\mathbf{B}}_{k-1}^{n} \mathbf{Q}_{k-1}\right\|_{\mathbf{Y} \rightarrow \mathbf{X}}=\left\|\tilde{\mathbf{B}}_{k-1}^{n} \mathbf{Q}_{k-1} \mathbf{T}\left(\mathbf{Q}_{k-1} \mathbf{T}\right)^{\dagger} \mathbf{Q}_{k-1}\right\|_{\mathbf{Y} \rightarrow \mathbf{X}} \leq \frac{1+c+\epsilon_{k-1}}{\underline{\gamma}_{k-1}}, \\
\left\|I-\tilde{\mathbf{S}}_{k} \mathbf{Q}_{k} \mathbf{T}\right\|_{\mathbf{X} \rightarrow \mathbf{X}} \leq 2\left(1+\frac{\overline{\bar{c}}}{\underline{\underline{c}}}\right)+\alpha_{k}, \quad\left\|\tilde{\mathbf{S}}_{k} \mathbf{Q}_{k} \mathbf{T}\right\|_{\mathbf{X} \rightarrow \mathbf{X}} \leq 1+\frac{\overline{\bar{c}}}{\underline{\underline{c}}}+\alpha_{k}
\end{gathered}
$$

(cf. (29), (30), (42), (43)). Imposing some maximal tolerance tol ${ }_{k} \in(0,1]$ on $\alpha_{k}$ and $\frac{\beta_{k}}{\underline{\gamma}_{k}}$,

$$
\alpha_{k} \leq \operatorname{tol}_{k}, \quad \frac{\beta_{k}}{\underline{\gamma}_{k}} \leq \operatorname{tol}_{k}, \quad k=0, \ldots, n,
$$

therewith implies an estimate of the form

$$
\begin{aligned}
\epsilon_{n} & \leq \epsilon_{0} \prod_{i=1}^{n}\left(1+C_{1} \operatorname{tol}_{i}\right)+C_{2} \sum_{j=1}^{n} \operatorname{tol}_{j} \prod_{i=j+1}^{n}\left(1+C_{1} \operatorname{tol}_{i}\right) \\
& \leq \epsilon_{0} \exp \left(C_{1} \sum_{j=1}^{n} \operatorname{tol}_{j}\right)+C_{2} \sum_{j=1}^{n} \operatorname{tol}_{j} \exp \left(C_{1} \sum_{i=j+1}^{n} \operatorname{tol}_{i}\right)
\end{aligned}
$$

with $C_{1}=3+2 \frac{\overline{\bar{C}}}{\underline{\underline{c}}}, C_{2}=(1+c)\left(3+2 \frac{\overline{\bar{C}}}{\underline{\underline{c}}}\right)$. In order to be able to conclude from (34) or (45) together with (49) and the estimate

$$
\left\|\tilde{\mathbf{B}}_{n}^{n} \mathbf{Q}_{n}\left(\tilde{\mathbf{T}}_{n}-\mathbf{T}\right) \mathbf{P}_{n}\right\|_{\mathbf{X} \rightarrow \mathbf{X}} \leq \frac{\left(1+c+\epsilon_{n}\right) \beta_{n}}{\underline{\gamma}_{n}}
$$

that also

$$
\left\|\left(I-\tilde{\mathbf{B}}_{n}^{n} \mathbf{Q}_{n} \tilde{\mathbf{T}}_{n}\right) \mathbf{P}_{n}\right\|_{\mathbf{X} \rightarrow \mathbf{X}} \leq \tilde{c}
$$

for some $\tilde{c} \in(0,1)$, we therefore demand the sum of all tolerances to be uniformly bounded with sufficiently small bound:

$$
\sum_{j=1}^{n} \operatorname{tol}_{j} \leq \bar{s}_{\mathrm{tol}}, \quad \operatorname{tol}_{n} \leq \overline{\mathrm{tol}}
$$


with $c+(1+c) \overline{\text { tol }}+(1+\overline{\operatorname{tol}})\left(\epsilon_{0}+C_{2} \bar{s}_{\text {tol }}\right) \exp \left(C_{1} \bar{s}_{\text {tol }}\right)<1$. In the context of subsection 3.1

$$
\alpha_{k}=\left\|\left(\tilde{\mathbf{T}}_{k}^{*}-\mathbf{T}^{*}\right) \mathbf{Q}_{k} \frac{1}{\xi_{k}} D_{k} \mathbf{Q}_{k} \mathbf{T}\right\|_{\mathbf{X} \rightarrow \mathbf{X}} \leq C h_{k}^{-p}\left\|\left(\tilde{\mathbf{T}}_{k}^{*}-\mathbf{T}^{*}\right) \mathbf{Q}_{k}\right\|_{\mathbf{Y} \rightarrow \mathbf{X}},
$$

and in subsection 3.2

$$
\alpha_{k}=\left\|\left(\tilde{\mathbf{T}}_{k}^{*}-\mathbf{T}^{*}\right) \mathbf{Q}_{k} c_{\xi} \hat{D}_{k} \mathbf{Q}_{k} \mathbf{T}\right\|_{\mathbf{X} \rightarrow \mathbf{X}} \leq C h_{k}^{-p}\left\|\left(\tilde{\mathbf{T}}_{k}^{*}-\mathbf{T}^{*}\right) \mathbf{Q}_{k}\right\|_{\mathbf{Y} \rightarrow \mathbf{X}},
$$

for some constant $C$ independent of $n$. Hence, in order to guarantee (48), (50), we demand the operator approximations $\tilde{\mathbf{T}}_{k}, \tilde{\mathbf{T}}_{k}^{*}$ to satisfy

$$
\begin{gathered}
h_{k}^{-p}\left\|\left(\tilde{\mathbf{T}}_{k}^{*}-\mathbf{T}^{*}\right) \mathbf{Q}_{k}\right\|_{\mathbf{Y} \rightarrow \mathbf{X}} \text { and } h_{k}^{-p}\left\|\mathbf{Q}_{k-1}\left(\tilde{\mathbf{T}}_{k}-\mathbf{T}\right)\right\| \mathbf{X} \rightarrow \mathbf{Y} \\
\text { summable with sufficiently small sum, }
\end{gathered}
$$

so that the approximation accuracy has to become higher with increasing level. For the symmetric multigrid operators and the additive one (46), one can do a similar perturbation analysis.

Remark 3. The exact implementation of projections $\mathbf{Q}_{j}$ involves inversion of Gramian matrices with respect to bases of $\mathbf{Y}_{n}$. Note that the system matrices in (8) are typically full so that the inversion of a tridiagonal matrix asymptotically does not count as compared to, e.g., a system matrix $\times$ vector multiplication. Nevertheless it might in some cases be advisable to save computational effort by using efficient numerical approximations $\tilde{\mathbf{Q}}_{j}$ of the projections $\mathbf{Q}_{j}$, that avoid Gram matrix inversions. Bramble, Pasciak, and Vassilevski [5] provide an abstract norm equivalence theorem (Theorem 2.1 in [5]) allowing for approximation operators $\tilde{\mathbf{Q}}_{j}$ to $\mathbf{Q}_{j}$ in (15) and give a concrete choice of $\tilde{\mathbf{Q}}_{j}$, based on nodal bases and local $L^{2}$-projections, that satisfies the assumptions of Theorem 2.1 in [5] in the context of piecewise linear finite elements $(r=0)$ and therefore can be used for an efficient inner product computation. For other implementations $\tilde{\mathbf{Q}}_{j}$ of $\mathbf{Q}_{j}$ that are based on wavelet-like space decompositions, see the references in [5].

\section{Full MUltigrid AS AN iterative Regularization MEthod}

The contraction factor estimates in Corollaries 2 [3] and 4 (that obviously also hold for cycles of more than one coarse grid correction) make it possible to apply the respective multigrid operators as preconditioners for (7), yielding level independent condition numbers (see [20] for an application of 46) as a preconditioner for a Newton-CG algorithm for the reconstruction of the reluctivity curve of nonlinearly magnetic materials).

Alternatively, the multigrid operators of Section 3 can be used in a full multigrid method (cf., e.g., [8]) for the iterative solution of (7):

Algorithm 5. (Full multigrid method)

Set $\tilde{x}_{0}:=\left(\mathbf{Q}_{0} T\right)^{\dagger} \mathbf{Q}_{0} y^{\delta}$

For $k=1, \ldots, n$

Set $\tilde{x}_{k}:=\tilde{x}_{k-1}+\mathbf{B}_{k}\left(\mathbf{Q}_{k} y^{\delta}-\mathbf{Q}_{k} \mathbf{T} \tilde{x}_{k-1}\right)$ which in matrix form with

$$
f_{j}^{k}:=\left\langle y^{\delta}, \psi_{j}^{k}\right\rangle_{\mathbf{Y}}, \quad j=1, \ldots, \operatorname{dim}\left(\mathbf{Y}_{k}\right), \quad k=0, \ldots n,
$$


reads as

Algorithm 6. (Full multigrid method in matrix form)

Set $\tilde{x}^{0}:=\left(\bar{M}^{0}\right)^{-1} f^{0}$

For $k=1, \ldots, n$

$$
\text { Set } \tilde{x}^{k}:=\left(\bar{C}^{k-1}\right)^{T} \tilde{x}^{k-1}+\overline{\mathbf{B}}^{k}\left(\bar{G}^{k}\right)^{-1}\left(f^{k}-\bar{M}^{k}\left(\bar{C}^{k-1}\right)^{T} \tilde{x}^{k-1}\right)
$$

Set $\tilde{x}_{n}:=\mathbf{T}^{*} \sum_{j=1}^{\operatorname{dim}\left(\mathbf{Y}_{n}\right)} \tilde{x}^{n} \psi_{j}^{n}$.

This iterative scheme can be easily shown to converge to an exact solution of the infinite-dimensional problem (11), if the data are exact, i.e., $\delta=0$. In the practically relevant situation of nonvanishing data noise, one has to take special care of the propagated data noise, though, which is amplified by a worst case factor $\sim h_{n}^{-p}$ at the $n$-th level, hence may explode as $n \rightarrow \infty$. It is therefore crucial to find for given data $y^{\delta}$ and noise level $\delta$ a stopping rule $n=N\left(\delta, y^{\delta}\right)$ for the iteration according to Algorithm 5, that carries out the trade-off between approximation error and propagated data noise in an optimal way in the sense that the so-defined approximation $\tilde{x}_{N\left(\delta, y^{\delta}\right)}$ converges to the exact best approximate solution of (1) as $\delta \rightarrow 0$, and additional a priori information (44) yields optimal rates (5) (cf. 18). It was shown in 21] that a Morozov-type discrepancy principle does so even without needing explicit knowledge of the exponent $\mu$ in (4).

Corollary 5. Let the assumptions of Corollary 2 or of Corollary 3 hold and fix $\tau>1$.

Then for any $\delta<\frac{\|y\|}{\tau+1}$ and for any data $y^{\delta}$ with (2), a finite $N\left(\delta, y^{\delta}\right)$, with

$$
N\left(\delta, y^{\delta}\right):=\min \left\{n \in \mathbb{N} \mid\left\|\mathbf{T} \tilde{x}_{n}-y^{\delta}\right\| \leq \tau \delta\right\}
$$

exists.

For any family of data $y^{\delta}$ with (2), $\tilde{x}_{N\left(\delta, y^{\delta}\right)}$ converges to $x^{\dagger}$ as $\delta \rightarrow 0$.

Under an additional source condition (44) for $\mu \leq \frac{1}{2}$, the optimal convergence rate (5) is achieved.

Proof. See the proof of Theorem 3 in [21].

Remark 4. To be able to treat also nonlinear ill-posed problems

$$
F(x)=y,
$$

one can either use the proposed multigrid operators in each linear step of a Newton iteration, or, analogously to [21, generalize the smoothing operators to directly incorporate them into a nonlinear multigrid method.

\section{NUMERICAL EXPERIMENTS}

For numerically verifying the theoretically predicted condition number estimates using the proposed multigrid operators as preconditioners, we implemented the proposed multigrid methods in a MatLab program on an SGI origin. As a simple application example we study the Abel integral equation

$$
\int_{0}^{t} \frac{x(s)}{\sqrt{t-s}} d s=y(t), \quad t \in(0,1),
$$

which represents the rotational symmetric two-dimensional case in X-ray tomography. Here $\mathbf{X}=\mathbf{Y}=L^{2}(0,1)$ and the degree of smoothing of the forward operator 


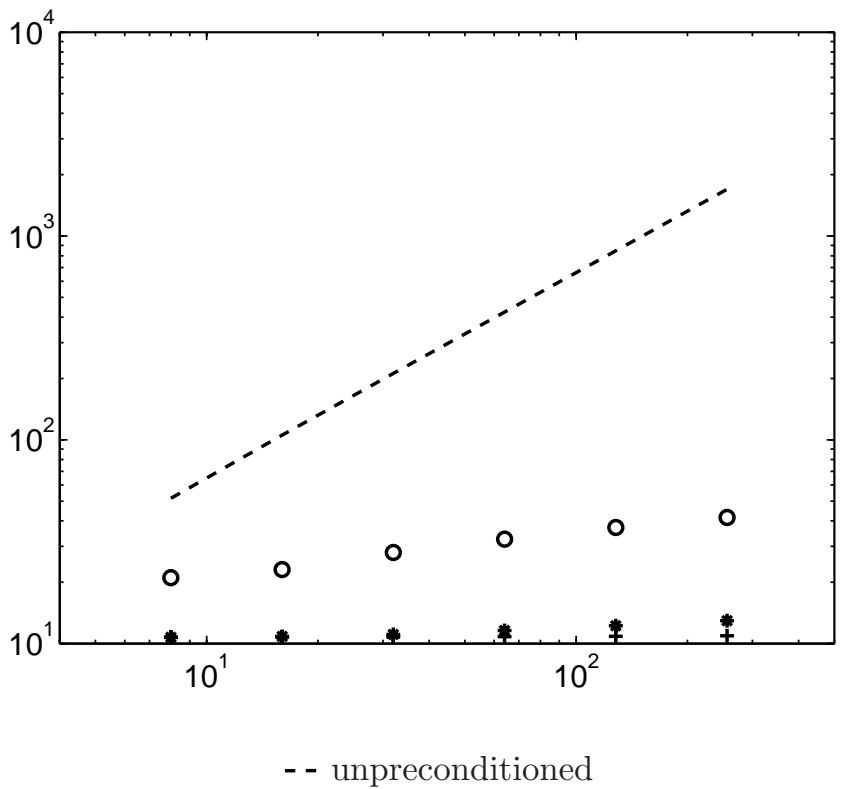

* Alg.3 preconditioner

+ Alg.4 preconditioner

- BPX preconditioner (46)

Figure 2. Condition numbers for Abel integral equation (52), projection onto piecewise linear $C^{0}$-splines.

and its adjoint

$$
\begin{aligned}
\mathbf{T}: \mathbf{X} & \rightarrow \mathbf{Y}, & \mathbf{T}^{*}: \mathbf{Y} & \rightarrow \mathbf{X} \\
x & \mapsto \int_{0}^{\cdot} \frac{x(s)}{\sqrt{-s}} d s, & & \mapsto \int_{.}^{1} \frac{y(t)}{\sqrt{t-.}} d t
\end{aligned}
$$

is $p=\frac{1}{2}$ (see, e.g., [10]; this can also be easily checked by taking Fourier transforms) and therefore $\mathbf{Y}^{-p}=H^{-\frac{1}{2}}(0,1), \mathbf{Y}^{-2 p}=H^{-1}(0,1)$, which corresponds to the situation considered in 3$]$. Using continuous piecewise linear splines for defining our discretization spaces $\mathbf{Y}_{n}$, we fulfill the norm equivalence requirements in both multiplicative multigrid variants of subsections 3.13 .2 as well as the additive one (46). The resulting condition numbers are plotted in Figure 2,

For further numerical experiments for a different model example we refer to [18], [19], [21]; a practical application example can be found in [20].

Remark 5. The theoretical analysis implies that the BPX preconditioner (46) and the modification of King's algorithm (according to Algorithm 4), which is just the multigrid algorithm resulting from applying the BPX preconditioner on each level as a smoother, give the same qualitative result. However, in our numerical experiments it turns out that the possible additional effort of implementing Algorithm 4 seems to pay by yielding better condition numbers. Moreover, the numerical tests show that the theoretically needed additional requirements (23), (24) for the generalization of Bramble, Leyk, Pasciak (according to Algorithm 3) do not seem to be really necessary (at least in our examples; see also [19]). 


\section{ACKNOWLEDGMENTS}

The author wishes to thank Dr. Schöberl from the SFB F013 in Linz for the hint at the important paper [3] as well as for interesting and instructive discussions on multigrid methods.

Moreover, we wish to acknowledge support by the Fonds zur Förderung der wissenschaftlichen Forschung under grant T 7-TEC and project F1308 within the Spezialforschungsbereich "Numerical and Symbolic Scientific Computing".

Finally, we want to thank the referee for his valuable comments on a first version of the manuscript.

\section{REFERENCES}

[1] D. Braess, W. Hackbusch, A new convergence proof for the multigrid method including the $V$-cycle, SIAM J. Numer. Anal. 20 (1983), 967-975. MR 85h:65233

[2] J.H. Bramble, Multigrid methods, Pitman Research Notes in Mathematics Series 294, Longman Scientific \& Technical, Harlow, UK, 1993. MR 95b:65002

[3] J.H. Bramble, Z. Leyk, J.E. Pasciak, The analysis of multigrid algorithms for pseudodifferential operators of order minus one, Math. Comp. 63 (1994), 461-478. MR 94m:65184

[4] J.H. Bramble, J.E. Pasciak, New estimates for multigrid algorithms including the $V$-cycle, Math. Comp. 60 (1993), 447-471. MR 94a:65064

[5] J.H. Bramble, J.E. Pasciak, P.S. Vassilevski, Computational scales of Sobolev norms with application to preconditioning, Math. Comp. 69 (2000), 463-480. MR 2000k:65088

[6] J.H. Bramble, J.E. Pasciak, J. Wang, J. Xu, Convergence estimates for multigrid algorithms without regularity assumptions, Math. Comp. 57 (1991), 23-45. MR 91m:65158

[7] J.H. Bramble, J.E. Pasciak, J. Xu, Parallel multilevel preconditioners, Math. Comp. 55 (1990), 1-22. MR 90k:65170

[8] A. Brandt, Multilevel adaptive solutions to boundary value problems, Math. Comp. 31 (1977), 333-390. MR 55:4714

[9] H.W. Engl, A. Neubauer, On projection methods for solving linear ill-posed problems, in: A. Vogel, ed., Model Optimization in Exploration Geophysics, Vieweg, Braunschweig, 1987, 73-92. MR 90g:65078

[10] H.W. Engl, M. Hanke, A. Neubauer, Regularization of Inverse Problems, Kluwer, Dordrecht, 1996. MR 97k:65145

[11] C.W. Groetsch, Inverse Problems in Mathematical Sciences, Vieweg, Braunschweig, 1993. MR 94m:00008

[12] C.W. Groetsch, A. Neubauer, Convergence of a general projection method for an operator equation of the first kind, Houston J. Mathem. 14 (1988), 201-207. MR 90b:65109

[13] Ch. Grossmann, H.-G. Roos, Numerik partieller Differentialgleichungen, Teubner, Stuttgart, 1994. MR 95i:65003

[14] W. Hackbusch, Multi-Grid Methods and Applications, Springer, Berlin, 1985. MR 87e:65082

[15] M. Hanke, C.R. Vogel, Two-level preconditioners for regularized inverse problems I: Theory, Numer. Math. 83 (1999), 385-402. MR 2001h:65069

[16] B. Kaltenbacher, A projection-regularized Newton method for nonlinear ill-posed problems with application to parameter identification problems with finite element discretization, SIAM J.Numer.Anal. 37 (2000), 1885-1908. MR 2001f:65070

[17] B. Kaltenbacher, Regularization by projection with a posteriori discretization level choice for linear and nonlinear ill-posed problems, Inverse Problems 16 (2000), 1523-1539. MR 2001h:65070

[18] B. Kaltenbacher, On the regularizing properties of a full multigrid method for ill-posed problems, Inverse Problems 17 (2001), 767-788. MR 2002h:65094

[19] B. Kaltenbacher, $V$-cycle convergence of some multigrid methods for ill-posed problems, SFB013-report, University of Linz, 2000.

[20] B. Kaltenbacher, M. Kaltenbacher, S. Reitzinger, Identification of nonlinear $B-H$ curves based on magnetic field computations and using multigrid preconditioners for ill-posed problems, to appear in Europ. J. Appl. Math., 2002. 
[21] B. Kaltenbacher, J. Schicho, A multigrid method with a priori and a posteriori level choice for the regularization of nonlinear ill-posed problems, Numer. Math., DOI 10.1007/s002110100375, January 2002.

[22] J.T. King, Multilevel algorithms for ill-posed problems, Numer. Math. 61 (1992), 311-334. MR 92k:65090

[23] A.K. Louis, Inverse und schlecht gestellte Probleme, Teubner, Stuttgart, 1989. MR 90g:65075

[24] V.A. Morozov, Regularization Methods for Ill-Posed Problems, CRC Press, Boca Raton, 1993. MR 94g:65002

[25] F. Natterer, Regularisierung schecht gestellter Probleme durch Projektionsverfahren, Numer. Math. 28 (1977), 329-341. MR 58:82:38

[26] F. Natterer, The Mathematics of Computerized Tomography, Teubner, Stuttgart, 1986. MR 88m: 44008

[27] P. Oswald, Multilevel Finite Element Approximations. Theory and Applications, Teubner, Stuttgart, 1994. MR 95k:65110

[28] S.V. Pereverzev, S. Prössdorf, On the characterization of self-regularization properties of a fully discrete projection method for Symm's integral equation, J. Integral Equations Appl. 12 (2000), no. 2, 113-130. MR 2001j:65198

[29] A. Rieder, A wavelet multilevel method for ill-posed problems stabilized by Tikhonov regularization, Numer. Math. 75 (1997), 501-522. MR 97k:65299

[30] A.N. Tikhonov, V.A. Arsenin, Methods for Solving Ill-Posed Problems, Nauka, Moskau, 1979. MR 82e: 65002

[31] G.M. Vainikko, U. Hämarik, Projection methods and self-regularization in ill-posed problems, Sov. Math. 29 (1985), 1-20. MR 87m:65096

[32] G.M. Vainikko, A.Y. Veterennikov, Iteration Procedures in Ill-Posed Problems, Nauka, Moscow, 1986. In Russian. MR 88c:47019

SFB013 Numerical and Symbolic Scientific Computing, University of Linz, FreitaedterStrasse 313, A-4040 Linz, Austria

E-mail address: barbara.kaltenbacher@sfb013.uni-linz.ac.at 